\title{
Comparison of the efficacy at 48 weeks of first-line antiretroviral treatment for HIV infection in 1998 and 2006: a multicentric investigation
}

\author{
F Sozio ${ }^{1}$, V Soddu ${ }^{2}$, G De Socio ${ }^{3}$, M D'Alessandro4 ${ }^{4}$ E Polilli1 ${ }^{1}$, G Madeddu², \\ P Bonfanti ${ }^{5}$, E Mazzotta ${ }^{1}$, J Vecchiet $^{4}$, MS Mura ${ }^{2}$, T Quirino $^{5}$, L Manzoli ${ }^{6}$ and \\ G Parruti*1
}

Address: ${ }^{1}$ Ospedale Civile Spirito Santo, Pescara, Italy, ${ }^{2}$ Department of Infectious Disease - University of Sassari, Sassari, Italy, ${ }^{3}$ Ospedale "Santa Maria della Misericordia", Perugia, Italy, ${ }^{4}$ Ospedale "SS. Annunziata" - University of Chieti, Chieti, Italy, ${ }^{5}$ Ospedale "L. Sacco", Milano, Italy and "Section of Epidemiology and Public Health, University of Chiety, Chieti, Italy

* Corresponding author

from Ninth International Congress on Drug Therapy in HIV Infection

Glasgow, UK. 9-13 November 2008

Published: 10 November 2008

Journal of the International AIDS Society 2008, I I (SuppI I):PI9 doi:I0.II86/I758-2652-II-SI-PI9

This abstract is available from: http://www.jiasociety.org/content/II/SI/PI9

(c) 2008 Sozio et al; licensee BioMed Central Ltd.

\section{Purpose of the study}

HAART efficacy for HIV-infected patients increased over time as more drugs and novel drug classes became available. It is not yet fully clear, however, which factors are most relevant to the increased success of more recent firstline regimens. We therefore planned to retrospectively investigate the efficacy of first-line regimens prescribed at our Institutions in 1998 and 2006.

\section{Methods}

Clinical records of all consecutive patients starting their first line HAART during 1998 and 2006 were evaluated for: age, sex, risk factors, co-morbidities, CDC stage, treatment adherence, record of any toxic event and/or therapy modification, basal, 24- and 48-week CD4 T-cell counts, HIV viremia, glycaemia, total cholesterol, triglycerides. All statistical analyses were performed using the Stata 10 software.

\section{Summary of results}

146 patients were included, $76.0 \%$ males, with a mean age of 38.2 years, mean basal CD 4 T-cells $250.3 / \mathrm{mm} 3$ and HIV viremia $5.66 \log 10$ copies/mL; 67 patients were considered in 1998, 79 in 2006, without any significant difference for basal values. HIV suppression at 48 weeks was observed in $59.1 \%$ of patients in 1998 and in $88.6 \%$ in 2006 ( $\mathrm{p}<0.001$ ). Multivariate logistic regression demonstrated that viral suppression was independently associated only with 48-week adherence (OR 20.6, CI 5.9-71.9, $\mathrm{p}<0.001$ ) and being treated in 2006 (OR 8.6, CI 2.4$30.6, \mathrm{p}<0.001)$. Moreover, adherence to HAART was significantly associated with better immune recovery at 24 weeks $(\mathrm{p}=0.01)$ and 48 weeks $(\mathrm{p}=0.02)$. Neither demographic, nor basal immune parameters, nor adverse events significantly altered the likelihood of viral suppression.

\section{Conclusion}

Our results indicate that after 48 weeks, patients treated with more recent first-line regimens have better chances of viral suppression and immune recovery than at the dawn of HAART. Adherence to therapy seems to be increased in parallel over time, and to be tightly associated with both outcomes. The independent association of better outcomes with the year of treatment and not with any other factor, including the incidence of adverse events, would suggest greater intrinsic potency of new HAART regimens. 